\title{
Acupuncture Therapies for Psoriasis Vulgaris: A Systematic Review of Randomized Controlled Trials
}

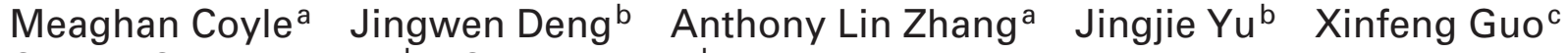 \\ Charlie Changli Xue ${ }^{a, b}$ Chuanjian Lu $^{\text {b }}$ \\ ${ }^{a}$ Traditional and Complementary Medicine Research Program, School of Health Sciences and Health Innovations Research Institute \\ (HIRi), RMIT University, Bundoora, Melbourne, VIC, Australia; \\ ${ }^{b}$ Department of Dermatology, Guangdong Provincial Hospital of Chinese Medicine (Guangdong Provincial Academy of Chinese \\ Medical Sciences), Guangzhou, China; \\ ${ }^{c}$ EBM and Clinical Research Service Group, Guangdong Provincial Hospital of Chinese Medicine (Guangdong Provincial Academy \\ of Chinese Medical Sciences), Guangzhou, China
}

\section{Keywords}

Acupuncture - Acupuncture therapy - Psoriasis .

Psoriasis vulgaris · Systematic review .

Randomized controlled trial

\section{Summary}

The purpose of this review was to evaluate the efficacy and safety of acupuncture therapies in the treatment of psoriasis vulgaris. Embase, CENTRAL, PubMed, AMED, CINAHL, CNKI, COVIP, CBM, and Wanfang databases were searched from inceptions to May 2013 for prospective randomized controlled trials evaluating acupuncture therapies for psoriasis vulgaris. No language limitations were applied. Studies were assessed using the Cochrane risk of bias tool. The primary outcome was Psoriasis Area Severity Index (PASI) score. Six studies (involving 522 participants) met the eligibility criteria for this review, and 5 were included in quantitative analysis. Due to the diversity of interventions, comparators and reported outcomes, meta-analysis was not possible. Results from single studies produced conflicting results for the outcomes PASI reduction, lesion reduction (non-PASI), PASI score, and relapse rate. There is some evidence of benefit of acupuncture therapies for the treatment of psoriasis vulgaris. However, the conclusions are limited by the small number of included trials and conflicting results from single studies. More research is needed to clarify the effect of acupuncture therapies for psoriasis vulgaris.

Meaghan Coyle and Jingwen Deng contributed equally to this paper.

\author{
Schlüsselwörter \\ Akupunktur · Akupunkturtherapie · Psoriasis . \\ Psoriasis vulgaris · Systematische Übersichtsarbeit . \\ Randomisierte kontrollierte Studie
}

\section{Zusammenfassung}

Ziel der Übersichtsarbeit war, die Effektivität und Sicherheit von Akupunkturtherapien bei der Behandlung von Schuppenflechte (Psoriasis vulgaris) zu untersuchen. Hierfür wurden die Datenbanken Embase, CENTRAL, PubMed, AMED, CINAHL, CNKI, COVIP, CBM und Wanfang nach prospektiven randomisierten kontrollieren Studien zur Akupunkturtherapie bei Schuppenflechte für den Zeitraum von Anfang bis Mai 2013 durchsucht. Die Auswahl erfolgte ohne Selektion der Sprache, in der die Studien durchgeführt worden waren. Die Studien wurden mittels des Cochrane-Instruments zur Erfassung des Bias-Risikos ausgewertet. Der primäre Endpunkt war der Psoriasis Area Severity Index (PASI)-Score. Sechs Studien ( $n=522$ Teilnehmer) erfüllten die Einschlusskriterien für dieses Review, 5 wurden in die quantitative Analyse eingeschlossen. Aufgrund der Vielfalt der Interventionen, der vergleichenden Instanzen und der berichteten Ergebnisse war eine Meta-Analyse nicht möglich. Die Ergebnisse einzelner Studien ergaben teils widersprüchliche Resultate für die PASI-Reduktion, die Reduktion von Verletzungen (nicht-PASI), den PASI-Score sowie die Rückfallquote. Obwohl es einige Belege zum Nutzen von Akupunkturtherapien bei Psoriasis vulgaris gibt, sind die Schlussfolgerungen, die aus unserer Erhebung gezogen werden können, durch die geringe Anzahl der eingeschlossenen Studien sowie die widersprüchlichen Ergebnisse der jeweiligen Untersuchungen beeinträchtigt. Weitere Forschung wird vonnöten sein, um die Wirkung von Akupunkturbehandlungen bei Psoriasis vulgaris zu prüfen.

\section{KARGER \\ Fax +497614520714

\section{() 2015 S. Karger GmbH, Freiburg}

$1661-4119 / 15 / 0222-0102 \$ 39.50 / 0$
Charlie Changli Xue, PhD

Traditional and Complementary Medicine Research Program

School of Health Sciences and Health Innovations Research Institute (HIRi), RMIT University

Bundoora, Melbourne, VIC, Australia

charlie.xue@rmit.edu.au 


\section{Introduction}

Psoriasis vulgaris is a common chronic inflammatory skin disorder, characterized by clearly delineated erythematous plaques which may be painful and pruritic [1]. The etiology and pathogenesis are not fully understood, but are thought to involve a hereditary component and environmental factors which trigger an inflammatory response, leading to hyperproliferation of keratinocytes $[1,2]$. The severity of psoriasis can vary over time, although remission is not common.

Worldwide psoriasis prevalence rates range from 0.1 to $3 \%$ [3]. Because of the appearance of the skin, psoriasis has a substantial impact on quality of life. Clinical practice guidelines suggest topical therapy, such as topical corticosteroids, vitamin D analogues, and topical retinoids for mild psoriasis $[4,5]$. Systemic therapy (retinoic acid, methotrexate, biological agents) is suggested for moderate to severe psoriasis [1]. These treatments may provide shortterm improvement, however, most of the therapies have serious adverse effects limiting their long-term use $[6,7]$.

Acupuncture is part of a broader family of techniques which stimulate acupuncture points to elicit a therapeutic effect [8]. The use of acupuncture can be traced back to more than 2000 years in China [9], and is gaining popularity in the USA and other parts of the Western world [10]. Other related therapies include electroacupuncture (an electrical stimulus applied to acupuncture needles), point application therapy (Chinese herbal medicine paste applied to acupuncture points, also known as acupoint sticking therapy), and bloodletting (puncture of a superficial blood vessel to release a small amount of blood).

Acupuncture directly involves contact with the skin, so it is not surprising that it has been used for the treatment of skin conditions in China for many years [11] with some benefit reported [12]. The use of CAM among patients with psoriasis has been explored in several studies, with a prevalence of 43-69\% [13-15]. Although the number of clinical trials using acupuncture to treat psoriasis is increasing, a comprehensive systematic review of acupuncture treatments for psoriasis has not been conducted so far. Thus, the purpose of this review was to determine the current state of evidence on acupuncture therapies for psoriasis vulgaris.

\section{Search Strategy and Selection Criteria}

PubMed, Embase, the Cochrane Central Register of Controlled Trials (CENTRAL), Cumulative Index to Nursing and Allied Health Literature (CINAHL), the Allied and Complementary Medicine Database (AMED), China National Knowledge Infrastructure (CNKI), Chongqing VIP Information Company (CQVIP), Chinese BioMedical Literature (CBM), and Wanfang databases were searched from inceptions to May 2013. No language limitations were applied. Free text and MeSH terms, including 'acupuncture', 'acupressure', 'acupoint', 'electroacupuncture', 'moxibustion', 'auricular therapy', 'psoriasis', 'psoriasi', and 'psoriases' were used.
Fig. 1. Risk of bias of included studies.

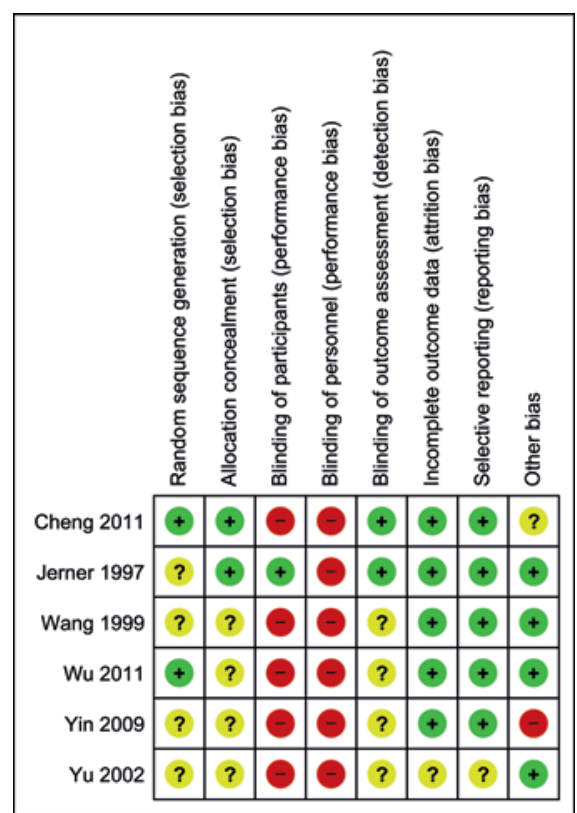

Studies were included if they were prospective randomized controlled trials (RCTs) reporting on the primary and secondary outcomes (see below). Eligible studies evaluated acupuncture therapies (either alone or in combination with other Chinese medicine (CM) methods of point stimulation) compared with sham or placebo acupuncture, Western medicine, or no treatment. Articles reporting on erythrodermic psoriasis, psoriatic arthritis, and guttate psoriasis were excluded, as were those combining acupuncture therapies with other forms of CM not involving point stimulation, e.g. Chinese herbal medicine. J.D. and J.Y. independently screened articles for inclusion. Disagreement was resolved by consulting a third author (M.C.). The primary outcome was Psoriasis Area and Severity Index (PASI) score reduction or lesion reduction, and secondary outcomes included PASI score, relapse rate, health-related quality of life, and adverse events.

\section{Data Extraction and Quality Assessment}

J.D. and J.Y. extracted the data independently. Data was extracted according to a standard template. We attempted to contact the original authors of the identified studies to obtain any missing data from the publications. Studies were categorized into predefined subgroups according to different PASI scores: either PASI $50 \geq$, or PASI $60 \geq$. A reduction in lesion severity of $50 \% \geq$ (PASI 50) is considered to be the minimum indicator of treatment effectiveness in international clinical practice guidelines [5]. However, the Chinese clinical practice guideline for psoriasis suggests PASI 60 as treatment goal [16], hence both levels of PASI reduction were included. We assessed the quality of included studies according to the Cochrane risk of bias domains (fig. 1). 


\section{Statistical Analysis}

For meta-analysis, we used Review Manager (RevMan) software (version 5.2). Dichotomous data were reported as risk ratio (RR) with $95 \%$ confidence intervals (CI), and continuous data were presented as mean difference (MD) and $95 \% \mathrm{CI}$. The estimates of the effect of the trials were computed using the random effects model, with intention-to-treat analysis used (where possible). We planned to assess statistical heterogeneity $\left(\mathrm{I}^{2}>50 \%\right)$ and publication bias, however as data was not able to be pooled this was not required.

\section{Results}

\section{Search Results}

The literature search retrieved 456 potentially relevant articles (fig. 2). After removal of duplicates, 268 articles remained. 238 were excluded through screening titles and abstracts, and a further 24 were excluded after review of full text. In the end, 6 studies involving 522 participants met the eligibility criteria for this review [17-22].

\section{Characteristics of Included Studies}

Characteristics of included studies are summarized in table 1 . One study was conducted in Sweden [18], and the remaining were conducted in China. All RCTs adopted a parallel-group design with 2 arms. The reported studies included 287 males and $237 \mathrm{fe}-$ males, although inconsistency was noted in the study by Yin and Zheng [20] where the total number of males and females $(\mathrm{N}=74)$ was greater than the number randomized patients $(\mathrm{N}=72)$. Age ranged from 27 [22] to 49 years [17]. One study recruited participants in the progressive stage of psoriasis [21] and 1 in the stable stage [17]. In the remaining studies, psoriasis stage was not specified. The sample size ranged from 54 [18] to 148 [22].

The included interventions were diverse (table 2), comprising bloodletting combined with cupping [17, 20], electroacupuncture [18], point application therapy [22], acupuncture plus moxibustion [19], and point injection (magnetic blood autoinfusion where the patients' blood was drawn, magnetized, and transfused back) [21]. Treatment duration ranged from 4 weeks [21, 22] to 12 weeks [19]. 3 studies included follow-up assessments after treatment had ceased $[17,18,22]$.

The average number of points used was 9 , although this varied from 1 [22] to 20 [18]. Overall, the most frequently used acupuncture points were Geshu BL17, Pishu BL20 (3 studies each), followed by Quchi LI11, Feishu BL13, Ganshu BL18, Xuechai SP10, and Dazhui GV14 (2 studies each). Geshu BL17 and Pishu BL20 were the acupuncture points most often selected as main points (3 studies each), and Quchi LI11 was the point most often selected as an additional point (an optional point added to a core set of points).

All studies reported on at least 1 of the specified outcomes. One study compared point injection of magnetic blood autoinfusion with intramuscular autoinfusion of non-magnetized blood [21]. The comparator included in the study is neither included in inter-

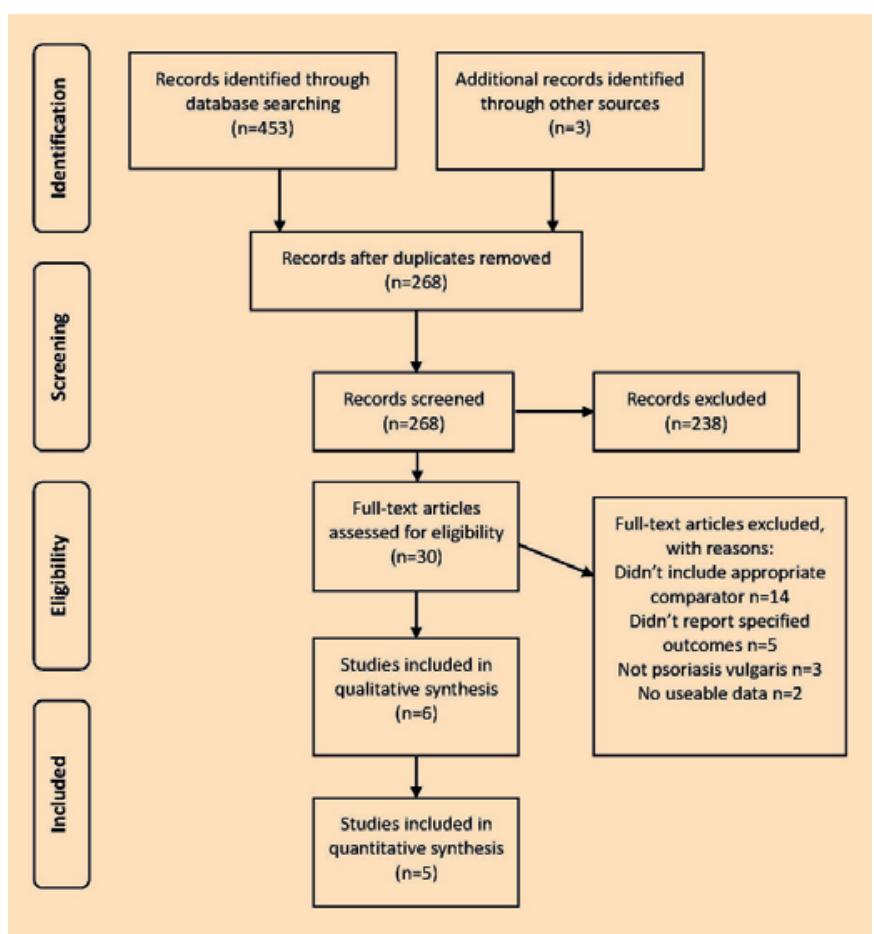

Fig. 2. Flowchart of trial selection.

nationally accepted clinical practice guidelines [5] nor in routine practice. As such, this study was excluded from quantitative analysis.

\section{Risk of Bias in Included Studies}

Assessments of risk of bias for included studies are presented in figure 1. All included trials were randomized. Two studies were assessed as low risk of bias for using appropriate methods for sequence generation $[17,19]$, and for the remaining 4 there was insufficient information to make a judgment of the level of risk. Allocation concealment was adequate in 2 studies (low risk of bias) $[17,18]$. One study was assessed as low risk of bias for adequately blinding participants to group allocation [18], while the remaining were assessed as high risk. All studies were assessed as high risk for blinding of personnel. Due to the nature of the intervention, it was impractical to blind therapists to group allocation. Two studies blinded outcome assessors and were assessed as low risk of bias $[17,18]$. The study by $\mathrm{Yu}[21]$ was assessed as unclear risk for incomplete outcome data and selective reporting while all other studies were assessed as low risk for these domains. There was insufficient information to assess other forms of bias, such as baseline imbalance or funding source [17]. The study by Yin and Zheng [20] contained reporting inconsistencies and was assessed as high risk for other bias. The remaining 4 studies were assessed as low risk of bias.

\section{Effect of Interventions}

Due to the diversity of interventions and comparators as well as differences in outcomes reported, it was not possible to pool results 


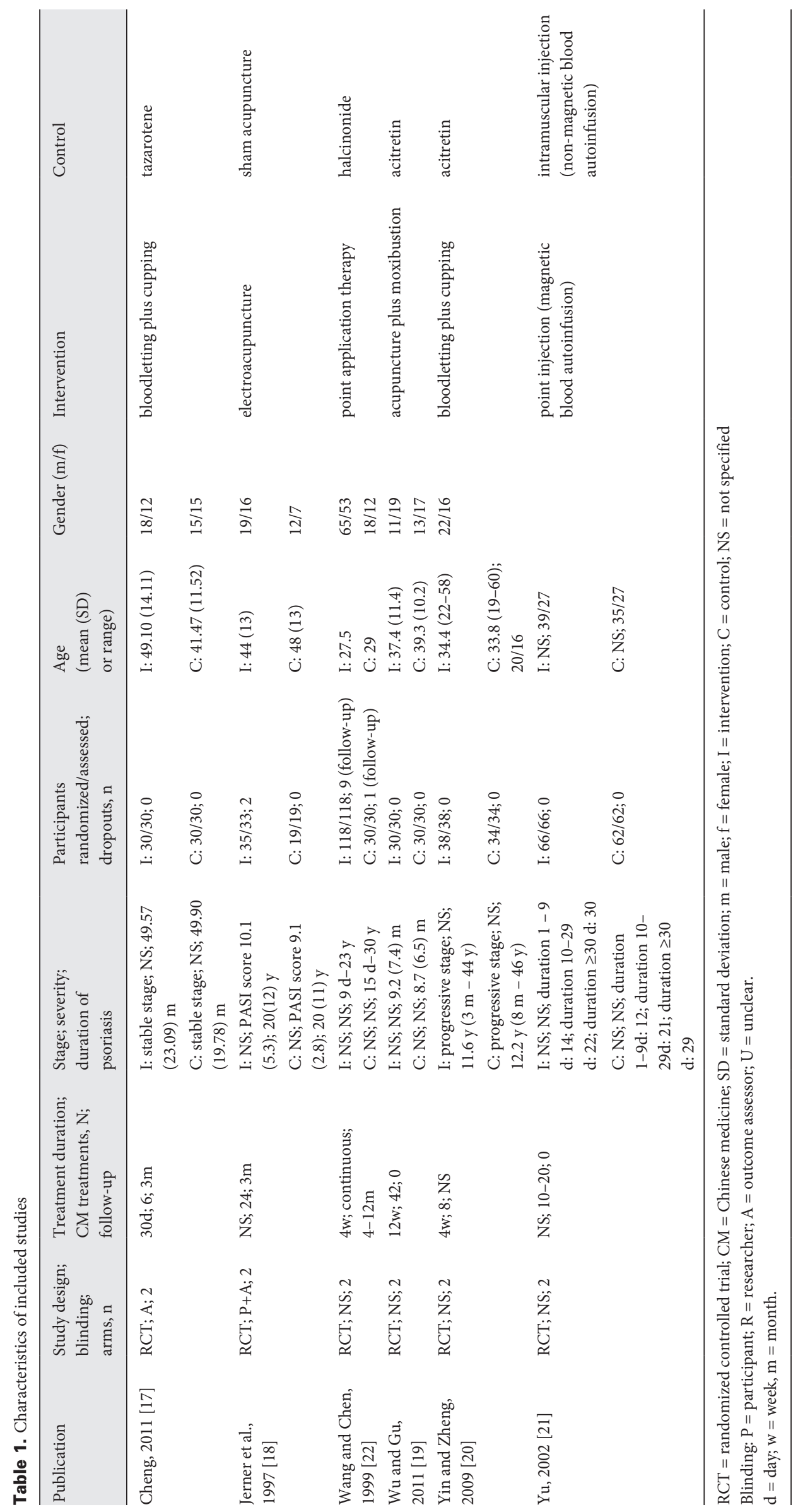




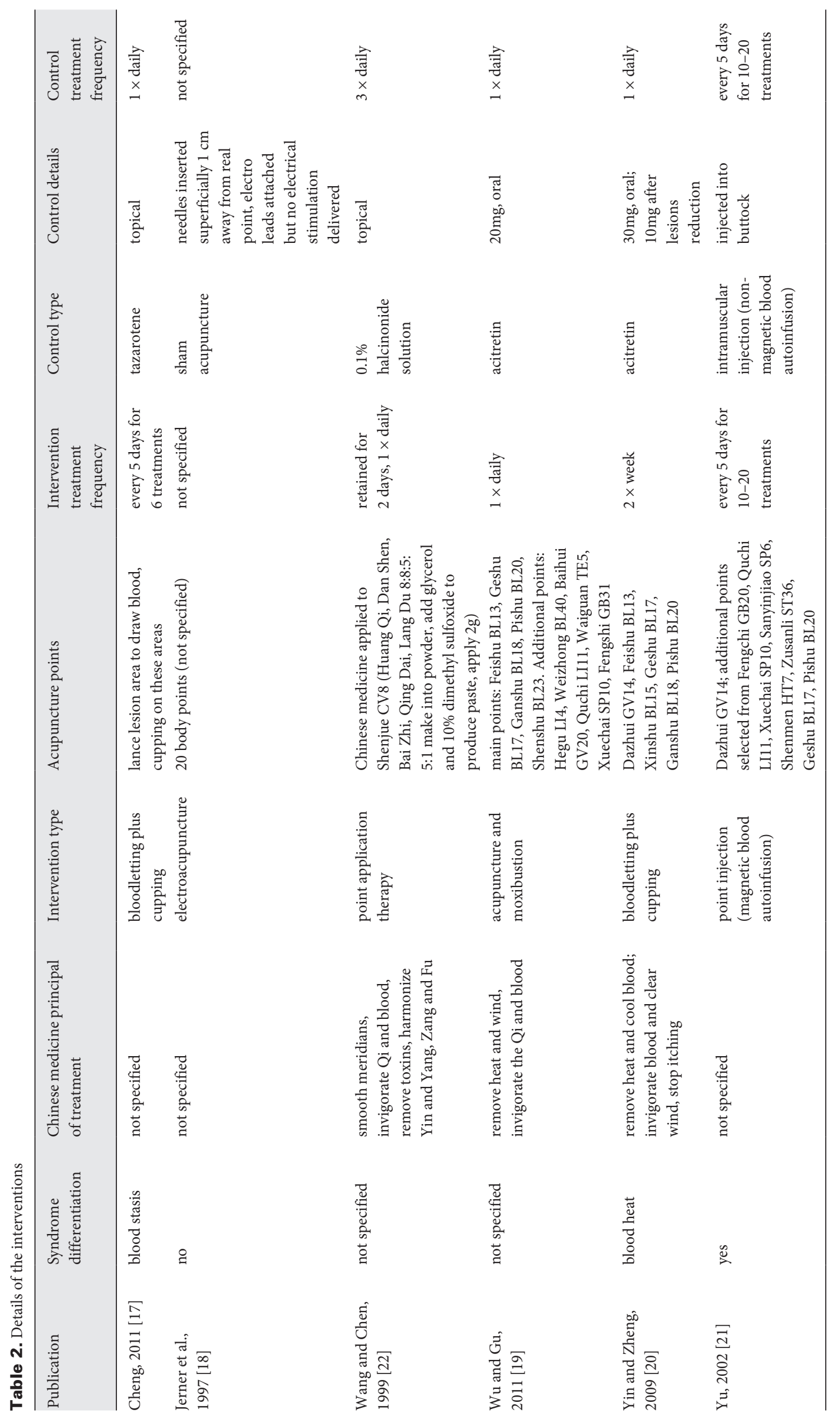


Table 3. Effects of interventions

\begin{tabular}{|c|c|c|c|c|}
\hline Comparison & Outcome & Studies & $\begin{array}{l}\text { Randomized/ } \\
\text { analyzed, } \mathrm{n}\end{array}$ & Effect estimate (95\% CI) \\
\hline $\begin{array}{l}\text { Acupuncture plus moxibustion } \\
\text { versus acitretin }\end{array}$ & PASI 60 & Wu and Gu, 2011 [19] & $60 / 60$ & $1.31^{* *}(0.87,1.97)$ \\
\hline $\begin{array}{l}\text { Point application therapy } \\
\text { versus halcinonide }\end{array}$ & $\begin{array}{l}\text { Lesion reduction (50\%) } \\
\text { Relapse rate (follow-up) }\end{array}$ & Wang and Chen, 1999 [22] & $\begin{array}{l}148 / 148 \\
148 / 138\end{array}$ & $\begin{array}{l}0.90^{* *}(0.79,1.02) \\
0.55^{* *}(0.33,1.00) \\
0.55^{* * *}(0.32,0.96)^{*}\end{array}$ \\
\hline $\begin{array}{l}\text { Bloodletting plus cupping } \\
\text { versus acitretin }\end{array}$ & PASI 60 & Yin and Zheng, 2009 [20] & $72 / 72$ & $1.85^{* *}(1.23,2.78)$ \\
\hline $\begin{array}{l}\text { Bloodletting plus cupping } \\
\text { versus tazarotene }\end{array}$ & $\begin{array}{l}\text { PASI } 50 \\
\text { Relapse rate (follow-up) } \\
\text { PASI score }\end{array}$ & Cheng, 2011 [17] & $\begin{array}{l}60 / 60 \\
60 / 42 \\
60 / 60\end{array}$ & $\begin{array}{l}1.47^{* *}(1.03,2.09)^{*} \\
0.23^{* *}(0.05,0.99)^{*} \\
-0.54^{* * * *}(-1.01,-0.07)^{*}\end{array}$ \\
\hline $\begin{array}{l}\text { Electroacupuncture versus } \\
\text { sham acupuncture }\end{array}$ & $\begin{array}{l}\text { PASI score } \\
\text { PASI score (follow-up) }\end{array}$ & Jerner et al, 1997 [18] & $\begin{array}{l}54 / 52 \\
54 / 52\end{array}$ & $\begin{array}{l}1.30^{* * * *}(-0.77,3.37) \\
0.70^{* * * *}(-0.71,2.11)\end{array}$ \\
\hline $\begin{array}{l}{ }^{*} \text { Statistically significant. } \\
{ }^{* *} \mathrm{RR}=\text { risk ratio; }{ }^{* * *} \mathrm{ITT}=\text { intent } \\
\text { a } \text { Only for those who achieved }\end{array}$ & ${ }^{* * * *} \mathrm{MD}=$ mean difference. & & & \\
\hline
\end{tabular}

from all studies. While 2 studies used acitretin as the comparator and reported on the outcome PASI $60[19,20]$, the interventions used were different and thus did not allow meta-analysis. As such, the effects of interventions below are presented according to comparisons. The results of analyses are included in table 3.

\section{Acupuncture plus Moxibustion versus Acitretin (1 Study)}

In 1 study $(\mathrm{N}=60)$, the number of people achieving PASI 60 who received acupuncture plus moxibustion was not less than of those who received acitretin [19].

\section{Point Application Therapy versus Halcinonide (1 Study)}

One study $(\mathrm{N}=148)$ found that point application therapy was not inferior to halcinonide in reducing lesion severity by $50 \%$, or in relapse rate [22]. However, when intention-to-treat analysis was performed to account for missing data on relapse rate, the result was in favor of halcinonide.

\section{Bloodletting plus Cupping versus Acitretin (1 Study)}

One study $(\mathrm{N}=72)$ found a greater number of people achieving PASI 60 in the bloodletting plus cupping group compared with those who received acitretin [20].

\section{Bloodletting plus Cupping versus Tazarotene (1 Study)}

In 60 participants, Cheng [17] found a greater number of people who achieved PASI 50 and a reduction in PASI score. In addition, a reduction in relapse rate at follow-up (for those who achieved PASI 50 at end of treatment) was also observed with bloodletting plus cupping treatment.

Electroacupuncture versus Sham Acupuncture (1 Study)

No benefit of electroacupuncture over sham acupuncture was found on PASI score at the end of treatment or at follow-up $(\mathrm{N}=$ 52) [18].

\section{Adverse Events}

Two studies reported on adverse events [17, 19]. Adverse events were dermatological in nature, and all adverse events were reported in participants allocated to the comparator group. Dry skin and skin burn was reported in 1 participant in the study by Cheng [17], while $\mathrm{Wu}$ and $\mathrm{Gu}$ [19] reported 10 people having dry skin, 7 cases of pruritus, and 6 cases of desquamation. No adverse events were reported among the acupuncture therapies in the included studies.

\section{Discussion}

The findings from this review show some promising evidence for acupuncture therapy in psoriasis, with an increasing number of people achieving clinically and statistically significant improvements. Specifically, improvements were observed for bloodletting plus cupping on PASI 50, PASI 60, and actua 1 PASI score. Additionally, lower relapse rates at follow-up were demonstrated. Further, no adverse events were reported in the treatment groups, suggesting that acupuncture and related therapies are well tolerated by participants.

The studies included in this review used a broad range of interventions, which overall reflects the diversity of CM and acupuncture practice. The comparator used also differed amongst studies, with only 2 studies using the same comparator $[19,20]$. This, in addition to differences in outcome measures, limited the ability to conduct meta-analyses, and the positive findings from this review were drawn from single studies. Therefore, the results of this review should be interpreted with caution. 
The range of interventions demonstrates the diversity in the treatment of psoriasis with acupuncture. However, there appears to be some commonality in the treatment approach and subsequently the acupuncture points stimulated across the studies. Of the 4 studies which reported the acupuncture points used, 3 reported using the points Geshu BL17, and Pishu BL20. The clinical actions of these points are to clear heat, and nourish blood [23]. In CM, heat and blood deficiency are seen as the cause of a variety of skin diseases. The actions of these points are aligned with treatment recommendations in CM clinical practice guidelines for treatment of psoriasis [16].

Only 2 of the 6 studies reported on adverse events [17, 19]. Future research must report on adverse events, including the severity of the events (even if none occurred), and assess the likelihood of relationship with the interventions. Koebner phenomenon (occurrence of psoriatic lesions where previously none existed after skin trauma [24], more commonly prevalent in unstable or flaring periods of psoriasis) was not reported in any of the studies although there are several case reports of Koebner phenomenon after needle acupuncture [25-27] and cupping therapy [28]. Acupuncture and related therapies should be used cautiously during the progressive stage of psoriasis when new lesions are developing, and patients should be advised of the risk of Koebner phenomenon.

None of the included studies reported on health-related quality of life. Psoriasis can have a significant impact on health-related quality of life [29-31]. The study by Gupta et al. [32] estimated depression and suicidal ideation being reported by more than $5 \%$ of people with psoriasis. Future research must include an assessment of health-related quality of life, such as the Dermatology Life Quality Index [33] and the Psoriasis Disability Index [34], to add evidence on the use of acupuncture therapies for psoriasis.

The cause of psoriasis still remains unclear - so does the mechanism of acupuncture in treating skin conditions [11]. Several theories have been proposed, including mechanisms involving the hypothalamus-pituitary-adrenal (HPA) axis, the autonomic nervous system, and brain derived neurotrophic factor [12]. Electroacupuncture may result in release of $\beta$-endorphin and corticotrophin into the peripheral blood [35] and lead to increased serum cortisol levels [36]. Activation of the hypothalamus and limbic system after acupuncture has been demonstrated on functional magnetic resonance imaging (fMRI) [37]. Further, acupuncture has been shown to decrease pruritic and inflammatory effects of histamine [38, 39]. Thus, more research is needed to explore the possible mechanisms of acupuncture in treating psoriasis.

The methodological quality of the included studies was low to average. Blinding of participants and personnel was assessed as high risk for all but 1 study [18], and only 2 studies reported blinded assessors $[17,18]$. Due to the nature of the interventions and comparators used in the included studies, it is challenging to achieve blinding of both participants and personnel when deliver- ing a manual therapy. Lack of blinding of participants and personnel has been shown to be associated with positive outcomes [40]. Despite the challenges, blinding of subjects and outcome assessors should be carried out to avoid the overestimation of effects observed in clinical trials that are not double-blind [41].

\section{Limitations of the Review}

As fewer than 10 studies were included in the review, it was not possible to investigate publication bias. Previous research has shown a possible relationship between countries, culture, and results [42]. Despite not being able to test for it, it is possible that publication bias exists amongst included studies, and the findings of this review should be interpreted accordingly.

As outlined above, the small number of included studies, in addition to small sample sizes, limits the interpretation of these findings. Inconsistencies were seen in the levels of treatment effect on PASI, with studies reporting PASI 95, PASI 70, PASI 60, PASI 50, and PASI 30. To demonstrate treatment effects, greater consistency is needed, in line with internationally accepted treatment goals and minimum lesion reduction level. There is also a need for high quality, scientifically rigorous RCTs of acupuncture therapies which report on relevant clinical outcomes as well as replication of these studies to further build the evidence base for acupuncture therapies for psoriasis.

\section{Conclusions}

The results of this systematic review suggest that evidence of acupuncture and related therapies for psoriasis vulgaris is promising. The results showed some benefit in improving PASI and decreasing relapse rate. However, these findings are limited by small sample sizes, the diversity of treatment interventions, and the small number of included studies. Acupuncture and related therapies do appear to be a safe treatment option for the treatment of psoriasis vulgaris, with no adverse events reported. Based on the results of this review, however, there is inadequate robust clinical evidence to establish acupuncture therapy as an effective treatment modality for psoriasis. Further research using scientifically rigorous study design with relevant clinical outcomes is needed.

\section{Disclosure Statement}

The research was partly funded by the following 2 projects: 1) International Science and Technology Cooperation Project of the Ministry of Science and Technology of China (012DFA31760); 2) Financial Industry Technology Research and Development Program of Guangdong Province of China (201105). The research was also supported by an International Research Grant from the Guangdong Provincial Academy of Chinese Medical Sciences, Guangdong Provincial Hospital of Chinese Medicine, China. 


\section{References}

1 Menter A, Gottlieb A, Feldman SR, et al.: Guidelines of care for the management of psoriasis and psoriatic arthritis: Section 1. Overview of psoriasis and guidelines of care for the treatment of psoriasis with biologics. J Am Acad Dermatol 2008:58:826-850.

$\checkmark 2$ Pathirana D, Ormerod AD, Saiag P, et al.: European S3-guidelines on the systemic treatment of psoriasis vulgaris. J Eur Acad Dermatol Venereol 2009;23(suppl 2):1-70.

3 Puig L, Bordas X, Carrascosa JM, et al.: Consensus document on the evaluation and treatment of moderate-to-severe psoriasis. Spanish psoriasis group of the Spanish Academy of Dermatology and Venereology (in Spanish). Actas Dermosifiliogr 2009;100:277-286.

4 Menter A, Korman NJ, Elmets CA, et al.: Guidelines of care for the management of psoriasis and psoriatic arthritis. Section 3. Guidelines of care for the management and treatment of psoriasis with topical therapies. J Am Acad Dermatol 2009;60:643-659.

5 Nast A., Boehncke WH, Mrowietz U, et al.: S3 Guidelines on the treatment of psoriasis vulgaris (English version). Update. J Dtsch Dermatol Ges 2012;10 (suppl 2):S1-95.

6 Al-Suwaidan SN, Feldman SR: Clearance is not a realistic expectation of psoriasis treatment. J Am Acad Dermatol 2000;42(5 Pt 1):796-802.

7 Naldi L, Svensson A, Diepgen T, et al.: Randomized clinical trials for psoriasis 1977-2000: the EDEN survey. J Invest Dermatol 2003;120:738-741.

8 World Health Organization Regional Office for the Western Pacific: WHO International Standard Terminologies on Traditional Medicine in the Western $\mathrm{Pa}$ cific Region. Manila, WPRO, 2007.

9 Wu JN: A short history of acupuncture. J Altern Complement Med 1996;2:19-21.

10 Barnes PM, Bloom B, Nahin RL: Complementary and alternative medicine use among adults and children: United States, 2007. National Health Statistics Reports 2008; 12. www.cdc.gov/nchs/data/nhsr/nhsr012.pdf (accessed March 2, 2015).

11 Tan EK, Millington GW, Levell NJ: Acupuncture in dermatology: an historical perspective. Int J Dermatol 2009;48:648-652.

12 Chen CJ, Yu HS: Acupuncture, electrostimulation, and reflex therapy in dermatology. Dermatol Ther 2003; 16: 87-92.

13 Baron SE, Goodwin RG, Nicolau N, et al.: Use of complementary medicine among outpatients with dermatologic conditions within Yorkshire and South Wales, United Kingdom. J Am Acad Dermatol 2005;52:589594.
4 Ben-Arye E, Ziv M, Frenkel M, et al.: Complementary medicine and psoriasis: linking the patient's outlook with evidence-based medicine. Dermatology 2003;207: 302-307.

15 Chen YF, Chang JS: Complementary and alternative medicine use among patients attending a hospital dermatology clinic in Taiwan. Int J Dermatol 2003;42: 616-621.

16 PLA Chinese Medicine Association Professional Committee of Dermatology: Consensus on integrative medicine diagnosis and treatment of psoriasis vulgaris. Chin J Dermatol Venereol Integr Tradit West Med 2009;5:328.

17 Cheng L: The clinical efficacy research on resting plaque psoriasis with prick bloodletting (in Chinese). Master thesis, Chengdu University of Traditional Chinese Medicine, 2011.

18 Jerner B, Skogh M, Vahlquist A: A controlled trial of acupuncture in psoriasis: no convincing effect. Acta Derm Venereol 1997;77:154-156.

19 Wu J-P, Gu S-Z: Randomized controlled trials for treatment of 30 cases of ordinary psoriasis by acupuncture and moxibustion (in Chinese). Zhen Ci Yan Jiu 2011;36:62-65.

20 Yin G, Zheng L: Clinical observation in 72 cases of blood-heat type psoriasis patients treated by blood-letting puncture (in Chinese). Xinjiang Journal of Traditional Chinese Medicine 2009;27:13-15.

21 Yu S: Clinical research in treating psoriasis by pointinjection of magnetic blood. International Journal of Clinical Acupuncture 2002;13:239-242.

22 Wang D, Chen J: Pingyinhu cream point application on CV8 for psoriasis vulgaris. Journal of External Therapy of Traditional Chinese Medicine 1999;8:4.

23 Maciocia G: The Foundations of Chinese Medicine. Edinburgh, Churchill Livingstone, 1989.

24 Weiss G, Shemer A, Trau H: The Koebner phenomenon: review of the literature. J Eur Acad Dermatol Venereol 2002;16:241-248.

25 Kirschbaum JO: Koebner phenomenon following acupuncture. Arch Dermatol 1972;106:767.

$26 \mathrm{Wu}$ JJ, Caperton C: Images in clinical medicine. Psoriasis flare from Koebner's phenomenon after acupuncture. N Engl J Med 2013;368:1635.

27 Zhu LL, Hong Y, Zhang L, et al.: Needle acupunctureinduced Koebner phenomenon in a psoriatic patient. J Altern Complement Med 2011;17:1097-1098.

28 Yu RX, Hui Y, Li CR: Koebner phenomenon induced by cupping therapy in a psoriasis patient. Dermatol Online J 2013; 19:18575.
9 Bhosle M, Kulkarni A, Feldman SR, et al.: Quality of life in patients with psoriasis. Health Qual Life Outcomes 2006;4:35

30 Krueger G, Koo J, Lebwohl M, et al.: The impact of psoriasis on quality of life: results of a 1998 National Psoriasis Foundation patient-membership survey. Arch Dermatol 2001;137:280-284.

31 Langley RG, Krueger GG, Griffiths CE: Psoriasis: epidemiology, clinical features, and quality of life. Ann Rheum Dis 2005;64(suppl 2):p. ii18-23; discussion ii24-5.

32 Gupta M, Schork NJ, Gupta AK, et al.: Suicidal ideation in psoriasis. Int J Dermatol 1993;32:188-190.

33 Finlay A, Khan G: Dermatology Quality of Life Index (DLQI): a simple, practical measure for routine clinical use. Clin Exp Dermatol 1994;19:210-216.

34 Finlay A, Kelly S: Psoriasis: an index of disability. Clin Exp Dermatol 1987;12:8-11.

35 Malizia E, Andreucci G, Paolucci D, et al.: Electroacupuncture and peripheral beta-endorphin and ACTH levels. Lancet 1979;2:535-536.

36 Lee SC, Yin SJ, Lee ML, et al.: Effects of acupuncture on serum cortisol level and dopamine beta-hydroxylase activity in normal Chinese. Am J Chin Med 1982; 1062-1069.

37 Wu MT, Hsieh JC, Xiong J, et al.: Central nervous pathway for acupuncture stimulation: localization of processing with functional MR imaging of the brain preliminary experience. Radiology 1999;212:133-141.

38 Lundeberg T, Bondesson L, Thomas M: Effect of acupuncture on experimentally induced itch. Br J Dermatol 1987;117:771-777.

39 Pfab F, Hammes M, Bäcker M, et al.: Preventive effect of acupuncture on histamine-induced itch: a blinded, randomized, placebo-controlled, crossover trial. J Allergy Clin Immunol 2005;116:1386-1388.

40 Ezzo J, Berman B, Hadhazy VA, et al.: Is acupuncture effective for the treatment of chronic pain? A systematic review. Pain 2000;86:217-225.

41 Schulz K, Chalmers I, Hayes RJ, Altman DG: Empirical evidence of bias. Dimensions of methodological quality associated with estimates of treatment effects in controlled trials. JAMA 1995;273:408-412.

42 Vickers A, Goyal N, Harland R, Rees R: Do certain countries produce only positive results? A systematic review of controlled trials. Control Clin Trials 1998;19: 159-166. 